\title{
Cholesterol-biosynthesis inhibitor stops infection
}

\begin{abstract}
The prevalence of multidrug-resistant bacterial strains has substantially increased over the past decade, and the lack of new classes of antibiotics has made the treatment of such infections increasingly challenging. Now, writing in Science, Oldfield and colleagues demonstrate that compounds originally designed to lower cholesterol levels in humans could have applications as effective virulence-factor-based therapies against methicillin-resistant Staphylococcus aureus (MRSA).

S. aureus is the leading cause of many human infections, particularly those of the skin and soft tissues.
\end{abstract} Moreover, MRSA - a variation of this bacterium that has evolved to resist $\beta$-lactam antibiotics, including methicillin - is responsible for thousands of deaths each year. An important virulence factor in S. aureus is the vivid golden carotenoid pigment staphyloxanthin, which acts as an antioxidant to evade attack by reactive oxygen species from the host immune system. Inhibition of staphyloxanthin biosynthesis is therefore an attractive potential therapeutic strategy to block bacterial infection.

Interestingly, earlier studies have noted that the process of staphyloxanthin production is strikingly similar to that of human cholesterol synthesis. Here, using X-ray crystallography, the authors analysed the structure of an essential enzyme involved in the first crucial step of staphyloxanthin biosynthesis: dehydrosqualene synthase (CrtM).
They cloned and purified CrtM from S. aureus and revealed that despite only modest sequence homology, it had close structural similarity to human squalene synthase (SQS). This led to the hypothesis that inhibitors of SQS that had already been developed as cholesterol-lowering drugs might also be active against CrtM.

To test their theory, they screened a selection of eight SQS inhibitors that were representative of the structural diversity of this group of agents. Three of them - the phosphonosulphonates - inhibited CrtM and subsequent staphyloxanthin formation. Further crystallographic analysis revealed the binding modes of these three compounds to CrtM.

Next, the authors assessed the antimicrobial potential of one of these phosphonosulphonates. BPH-652 was selected as the test compound, as it strongly inhibited staphyloxanthin formation and had already progressed into early human clinical trials as a cholesterol-lowering agent.

Together with the production of white $S$. aureus, which signifies the absence of the staphyloxanthin antioxidant, BPH-652 dramatically decreased $S$. aureus survival. Specifically, it demonstrated a 15 -fold increased susceptibility to death of S. aureus by hydrogen peroxide and 4-fold decreased survival ability in freshly isolated human whole blood. Furthermore, in a mouse model of systemic $S$. aureus infection, BPH-652 treatment decreased

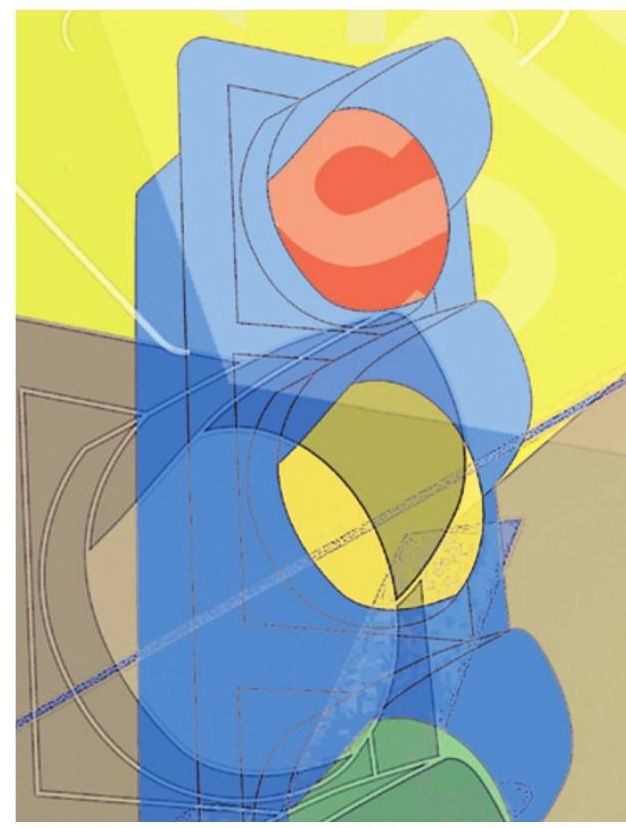

bacterial counts in the kidney by $98 \%$, achieving almost undetectable levels. Importantly, and consistent with the low toxicity profile observed in previous clinical trials, $\mathrm{BPH}-652$ had no effect on the growth of the various human cell lines tested.

These data suggest that phosphonosulphonate cholesterol-lowering agents provide a promising lead for the development of a novel and safe anti-infective treatment against MRSA. Such virulence-factor-based approaches offer a new direction for therapies targeting multidrug-resistant bacterial strains, and may theoretically reduce the selection pressure towards the emergence of resistance.

\section{Sarah Crunkhorn}

ORIGINAL RESEARCH PAPER Liu, C.-I. et al. A cholesterol biosynthesis inhibitor blocks Staphylococcus aureus virulence. Science 14 Feb 2008 (doi:10.1126/science.1153018) FURTHER READING Liu, G. et al. Staphylococcus aureus golden pigment impairs neutrophil killing and promotes virulence through its antioxidant activity. J. Exp. Med. 202, 209-215 (2005) 